TRANSACTIONS OF THE

AMERICAN MATHEMATICAL SOCIETY

Volume 173, November 1972

\title{
A NEW CLASS OF FUNCTIONS OF BOUNDED INDEX
}

BY

\author{
S. M. SHAH( $\left.{ }^{1}\right)$ AND S. N. SHAH( ${ }^{2}$ )
}

ABSTRACT. Entire functions of strongly bounded index have been defined and it is shown that functions of genus zero and having all negative zeros satisfying a one sided growth condition belong to this class.

1. Introduction. Let $f(z)$ be an entire function and let

$$
\Omega(z)=\Omega_{s}(z)=\max _{0 \leq j \leq s}\left\{\frac{\left|f^{(j)}(z)\right|}{j !}\right\} \quad\left(f^{(0)}(z)=f(z)\right) .
$$

Definition 1. An entire function $f(z)$ is said to be of bounded index (we shall also say of class $B$ ), if for some fixed $s,\left\{\left|f^{(n)}(z)\right| / n !\right\} \leq \Omega_{s}(z)$ for all $n$ and all $z$ (see [3], [6]).

It is known that given any transcendental entire function $f$, there exists a transcendental entire function $g$ of unbounded index such that [7]

$$
\log M(r, f) \sim \log M(r, g) \quad(r \rightarrow \infty) .
$$

In particular, given two numbers $\lambda$ and $\rho$ such that $0 \leq \lambda \leq \rho \leq \infty$, there exists a function $g$ of unbounded index such that $g$ is of order $\rho$ and of lower order $\lambda$. A result of this type cannot hold with $g$ of bounded index since a function of bounded index must necessarily be of exponential type [8]. Furthermore, known examples of functions of bounded index and order one are all of regular growth, that is, the order of a function is equal to its lower order ([5], [9]). In this paper we show that there exist functions of bounded index, and of given order $\rho$ and lower order $\lambda$ provided $0 \leq \lambda \leq \rho \leq 1$ (see also [10]). Our attempts to construct such functions have led us to the remark that a very simple subclass $S B$, of the class $B$, displays a particularly useful property. If $f \in S B$ and $P$ is a polynomial then $f P \in S B$.

Definition 2. An entire function $f(z)$ is of strongly bounded index (we shall also say of class $S B$ ) if there exist quantities $\chi, 0<\chi<1, r_{0}$, and an integer $s \geq 0$ such that

Presented to the Society, March 5, 1971 under the title Entire functions of strongly bounded index and March 31, 1971 under the title Entire functions of strongly bounded index. II; received by the editors October 4, 1971.

AMS (MOS) subject classifications (1970). Primary 30A64; Secondary 30A66.

Key words and phrases. Order and lower order of an entire function, exponential type, bounded index.

(1) Research supported by N.S.F. Grant GP-19533.

(2) Research supported by N.S.F. Grant GP-7507.

Copyright @ (1973, American Mathematical Society 


$$
\left|f^{(n)}(z)\right| / n ! \leq \chi \Omega_{s}(z),
$$

for all $n \geq s+1$ and all $z$ with $|z| \geq r_{0}$. For instance, $f(z)=e^{z} \in S B$. Here $X=1 / 2, s=1, r_{0}=0$. We now state

Theorem 1. Let $f(z)$ be entire and $f(z) \in S B$. Then

(i) $f(z) \in B$,

(ii) if $P(z)$ is a polynomial then $f(z) P(z) \in S B$,

(iii) $\{f(z) / P(z)\} \in S B$ provided $\{f(z) / P(z)\}$ is entire,

(iv) if $a$ is any complex number and

$$
0<\chi<e^{-2|a|}
$$

where $\chi$ is the constant in (1.2), then $e^{a z} f(z) \in S B$.

Our main result is

Theorem 2. Let $\left\{a_{n}\right\}_{n=1}^{\infty}$ be a positive, strictly increasing sequence sucb that

$$
a_{n+1}-a_{n} \geq b_{n} \quad(n \geq 1),
$$

where $\left\{b_{n}\right\}_{n=1}^{\infty}$ is positive nondecreasing and

$$
\sum_{n=1}^{\infty} \frac{1}{n b_{n}}<\infty
$$

Then

$$
f(z)=\prod_{n=1}^{\infty}\left(1+\frac{z}{a_{n}}\right) \in S B .
$$

Theorem 2 has four useful corollaries. Consider the Lindelöf functions

$$
f(z)=\prod_{1}^{\infty}\left(1+\frac{z}{a_{n}}\right),
$$

where $a_{n}=\left\{n(\log n)^{a}\right\}^{1 / \lambda}, 0<\lambda \leq 1$ and $\alpha>1$ if $\lambda=1, a$ an arbitrary real number if $0<\lambda<1$.

Corollary 2.1. All Lindelöf functions defined by (1.7) belong to class SB.

Corollary 2.2. If $a$ is any nonzero complex number and $f(z)$ satisfies the assumptions of Theorem 2, then

$$
f(a z)=F(z) \in S B .
$$

Corollary 2.3. Let $\left\{a_{n}\right\}_{n=1}^{\infty}$ satisfy the conditions of Theorem 2, and let $\left\{a_{n_{j}}\right\}_{j=1}^{\infty}$ be any one of its infinite subsequences. 
Then

$$
\prod_{j=1}^{\infty}\left(1+\frac{z}{a_{n_{j}}}\right)=g(z) \in S B .
$$

From Corollary 2.2, we deduce that $F(z) \in S B \subset B$ and consequently there exists an index $s$ such that

$$
\frac{\left|F^{(n)}(z)\right|}{n !} \leq \max _{0 \leq j \leq s}\left\{\frac{\left|F^{(j)}(z)\right|}{j !}\right\},
$$

for all $n$ and all $z$.

Assume now $a$ real and greater than one so that (1.8) and (1.1) imply

$$
\frac{a^{n}\left|f^{(n)}(\alpha z)\right|}{n !} \leq \alpha^{s} \Omega_{s}(\alpha z)
$$

Replacing $\alpha z$ by $\zeta$, we obtain

$$
\left|f^{(n)}(\zeta)\right| / n ! \leq \Omega_{s}(\zeta) / \alpha^{n-s} \quad(n=s+1, s+2, \ldots)
$$

for all $\zeta$.

We thus see that the functions in Theorem 2 belong to $S B$ in a very special sense: the constant $\chi$ in (1.2) may be chosen arbitrarily small (a diminution of $\chi$ will of course increase, in general, the value of the index $s$ ).

In particular, if $a$ is given, we can choose $\chi$ so as to satisfy (1.3). Consequently assertion (iv) of Theorem 1 leads to

Corollary 2.4. If $f(z)$ satisfies the conditions of Theorem 2 then $e^{a z+b} f(z)$ $\epsilon S B$.

In Corollary 2.3 we can choose a subsequence $\left\{a_{n_{j}}\right\}$ by omitting from the given sequence $\left\{a_{n}\right\}$ long sections of consecutive terms. The entire function $b(z)$ corresponding to this subsequence belongs the the class $S B$ and it is obvious that we may, by suitable choices of the gaps, obtain irregularities in the growth of $b(z)$. We are thus led to the following result which we state without proof.

Theorem 3. Let $f(z)$ be given by (1.6) and let a sequence $\left\{a_{n}\right\}_{n=1}^{\infty}$ satisfy the conditions of Theorem 2. Let

$$
R_{1}, R_{2}, \cdots \quad\left(R_{m}<R_{m+1}, m=1,2, \ldots, R_{m} \rightarrow \infty\right)
$$

be a given sequence.

It is always possible to select a subsequence $\left\{c_{j}\right\}_{j=1}^{\infty}$ of $\left\{a_{n}\right\}_{n=1}^{\infty}$ and two subsequences $\left\{R_{k}^{\prime}\right\}_{k=1}^{\infty},\left\{R_{k}^{\prime \prime}\right\}_{k=1}^{\infty}$ of (1.9) sucb that

$$
b(z)=\prod_{j=1}^{\infty}\left(1+\frac{z}{c_{j}}\right) \in S B,
$$


and sucb that for all $k=1,2, \ldots$

$$
\log M\left(R_{k}^{\prime}, b\right)>(1-1 /(k+1)) \log M\left(R_{k}^{\prime}, f\right),
$$

and

$$
\frac{\log \log M\left(R_{k}^{\prime \prime}, b\right)}{\log R_{k}^{\prime \prime}}<\frac{1}{k+1} .
$$

By an appropriate choice of gaps we can also construct a function $b$ belonging to $B$ and of given order $\rho$ and of given lower order $\lambda$ where $0 \leq \lambda \leq \rho \leq 1$. We omit the details of construction.

In $\$ 2$ we give the proof of Theorem $1 . \$ 3$ contains necessary lemmas and $\$ 4$ gives the proof of Theorem 2 .

The authors wish to thank Professor Albert Edrei who suggested the consideration of functions of the class $S B$ and conjectured Theorems 1 and 2 .

2. Proof of Theorem 1. Proof of assertion (i). By Definition 2, there exist fixed quantities $\chi, 0<\chi<1, r_{0}$ and $s \geq 0$ such that (1.2) holds for all $n \geq s+1$ and all $z$ with $|z| \geq r_{0}$.

We examine $f(z)$ and its successive derivatives in the closed disk

$$
|z| \leq r_{0}
$$

Since the number of zeros of $f$ in $(2.1)$ is $n\left(r_{0}, 1 / f\right)$, it is obvious that one of the quantities $f(z), f^{\prime}(z), \ldots, f^{(N)}(z)\left(N=n\left(r_{0}, 1 / f\right)\right)$ is different from zero.

Let

$$
\Omega_{N}(z)=\max _{0 \leq j \leq N}\left\{\frac{\left|f^{(j)}(z)\right|}{j !}\right\} .
$$

It is clear that $\Omega_{N}(z)$ is continuous and does not vanish in (2.1). Hence for some $\alpha>0$,

$$
\Omega_{N}(z) \geq \alpha \quad\left(|z| \leq r_{0}\right) .
$$

Assume $|z| \leq r_{0}$. By Cauchy's formula, for the $n$th derivative,

$$
\frac{\left|f^{(n)}(z)\right|}{n !} \leq \frac{1}{2^{n}} M\left(r_{0}+2, f\right) \text {. }
$$

If $n$ is sufficiently large, say $n \geq n_{0} \geq s+1,(2.3)$ and (2.4) imply

$$
\frac{\left|f^{(n)}(z)\right|}{n !} \leq \frac{M\left(r_{0}+2, f\right)}{2^{n}} \leq \chi^{\alpha} \leq \chi \Omega_{N}(z)
$$

for all $z$ such that $|z| \leq r_{0}$ and for all $n \geq n_{0}$. Let $p=\max \left(n_{0}, N\right)$. Then (2.5) and (2.2) imply 


$$
\frac{\left|f^{(n)}(z)\right|}{n !} \leq x \max _{0 \leq j \leq p}\left\{\frac{\left|f^{(j)}(z)\right|}{j !}\right\} \quad(n \geq p+1),
$$

provided $|z| \leq r_{0}$. On the other hand, since $n_{0} \geq s+1$, and $f \in S B,(2.6)$ holds for $n \geq p+1$ and $|z| \geq r_{0}$. Hence we can drop the restriction on the size of $|z|$ and this completes the proof.

Proof of assertion (ii). By hypothesis (1.2) holds for all $n \geq s+1$ and all $z$ such that $|z| \geq r_{0}$. Let

$$
\begin{gathered}
g(z)=\left(z-z_{0}\right) f(z), \\
\Omega(z)=\max _{0 \leq j \leq s}\left\{\frac{\left|f^{(j)}(z)\right|}{j !}\right\}, \\
\Omega^{*}(z)=\max _{0 \leq j \leq s+1}\left\{\frac{\left|g^{(j)}(z)\right|}{j !}\right\} .
\end{gathered}
$$

Since

$$
\frac{g^{(n)}(z)}{n !}=\left(z-z_{0}\right) \frac{f^{(n)}(z)}{n !}+\frac{f^{(n-1)}(z)}{(n-1) !}
$$

we have, for $n \geq s+2$ and $|z| \geq r_{0}$,

$$
\frac{\left|g^{(n)}(z)\right|}{n !} \leq \chi \Omega(z)\left\{1+\left|z-z_{0}\right|\right\}
$$

From (2.7) we obtain, for $z \neq z_{0}$,

$$
\frac{f^{(n)}(z)}{n !}=\frac{g^{(n)}(z)}{n !} \frac{1}{\left(z-z_{0}\right)}+\frac{g^{(n-1)}(z)}{1 !(n-1) !} \frac{(-1) 1 !}{\left(z-z_{0}\right)^{2}}+\cdots+\frac{g(z)}{0 !} \frac{(-1)^{n} n !}{n !\left(z-z_{0}\right)^{n+1}}
$$

(2.9) and (2.12) yield, for $z \neq z_{0}$,

$$
\frac{\left|f^{(n)}(z)\right|}{n !} \leq \Omega^{*}(z)\left\{\frac{1}{\left|z-z_{0}\right|}+\frac{1}{\left|z-z_{0}\right|^{2}}+\cdots+\frac{1}{\left|z-z_{0}\right|^{n+1}}\right\} \quad(0 \leq n \leq s+1) .
$$

Consequently we have from (2.11) and (2.8), for $n \geq s+2$ and $|z| \geq r_{0}, z \neq z_{0}$,

$$
\frac{\left|g^{(n)}(z)\right|}{n !} \leq \chi \Omega^{*}(z)\left\{1+\left|z-z_{0}\right|\right\}\left\{\frac{1}{\left|z-z_{0}\right|}+\cdots+\frac{1}{\left|z-z_{0}\right|^{n+1}}\right\} \text {. }
$$

If $|z|$ is sufficiently large, say $|z| \geq R_{1}$, then

$$
\chi\left\{1+\left|z-z_{0}\right|\right\}\left\{\frac{1}{\left|z-z_{0}\right|}+\cdots+\frac{1}{\left|z-z_{0}\right|^{n+1}}\right\}<\chi^{\prime}
$$

where $0<\chi^{\prime}<1$. This shows that $g(z) \in S B$. Now if $P(z)=\left(z-z_{0}\right) \cdots\left(z-z_{p}\right)$ 
and $Q(z)=A P(z)(A$ a constant $)$ then the above argument applied $(p+1)$ times shows that $f P \in S B, f Q \in S B$. This completes the proof.

Proof of assertion (iii). Let

$$
g(z)=f(z) /\left(z-z_{0}\right)
$$

and let $\Omega(z)$ and $\Omega^{*}(z)$ have the same meaning as in (2.8) and (2.9). Then for $n \geq s+1$ and $|z| \geq r_{0}, z \neq z_{0}$,

$$
\frac{\left|g^{(n)}(z)\right|}{n !} \leq \Omega(z)\left\{\frac{\chi}{\left|z-z_{0}\right|}+\frac{1}{\left|z-z_{0}\right|^{2}}+\cdots+\frac{1}{\left|z-z_{0}\right|^{n+1}}\right\} .
$$

From (2.13) we have, for $1 \leq n \leq s$,

$$
\frac{\left|f^{(n)}(z)\right|}{n !} \leq \frac{\left|g^{(n)}(z)\right|}{n !}\left|z-z_{0}\right|+\frac{\left|g^{(n-1)}(z)\right|}{(n-1) !} \leq \Omega^{*}(z)\left\{1+\left|z-z_{0}\right|\right\}
$$

and

$$
|f(z)|=|g(z)|\left|z-z_{0}\right| \leq \Omega^{*}(z)\left\{1+\left|z-z_{0}\right|\right\} .
$$

Hence (2.15) holds for $0 \leq n \leq s$ and

$$
\Omega(z) \leq \Omega^{*}(z)\left\{1+\left|z-z_{0}\right|\right\} .
$$

The inequalities (2.14) and (2.16) imply

$$
\frac{\left|g^{(n)}(z)\right|}{n !} \leq \Omega^{*}(z)\left\{1+\left|z-z_{0}\right|\right\}\left\{\frac{\chi}{\left|z-z_{0}\right|}+\cdots+\frac{1}{\left|z-z_{0}\right|^{n+1}}\right\}
$$

for $n \geq s+1$ and $|z| \geq r_{0}, z \neq z_{0}$. Hence for $|z|$ sufficiently large, say $|z| \geq R_{2}$, we have $\left|g^{(n)}(z)\right| / n ! \leq \chi^{\prime \prime} \Omega^{*}(z)$, where $0<\chi^{\prime \prime}<1$, for $n \geq s+1$ and $|z| \geq R_{2}$. This means that $g(z) \in S B$.

If $P(z)=\Pi_{j=0}^{p}\left(z-z_{j}\right)$ and $Q(z)=A P(z)$, then the above argument shows that $f / P \in S B, f / Q \in S B$. This completes the proof.

Proof of assertion (iv). Let

$$
g(z)=e^{a z} f(z) .
$$

Then

$$
\frac{g^{(n)}(z)}{n !}=e^{a z}\left\{\frac{f^{(n)}(z)}{n !}+a \frac{f^{(n-1)}(z)}{(n-1) !}+\cdots+\frac{a^{n}}{n !} f(z)\right\} .
$$

There is a similar relation where $f$ and $g$ are exchanged and $a$ is replaced by - a. From this latter formula we deduce 
In particular if

$$
\frac{\left|f^{(n)}(z)\right|}{n !} \leq\left|e^{-a z}\right| \max _{0 \leq j \leq n}\left\{\frac{\left|g^{(j)}(z)\right|}{j !}\right\} e^{|a|} .
$$

$$
\Omega^{* *}(z)=\max _{0 \leq j \leq s}\left\{\frac{\left|g^{(j)}(z)\right|}{j !}\right\},
$$

we have, in view of (1.1),

$$
\Omega(z) \leq\left|e^{-a z}\right| e^{|a|} \Omega^{* *}(z) .
$$

By assumption, (1.2) is satisfied for some fixed $\chi<1$ and consequently (2.19) yields for all $n \geq s+1$ and all $z$ such that $|z| \geq r_{0}$

$$
\begin{aligned}
\frac{\left|g^{(n)}(z)\right|}{n !} & \leq\left|e^{a z}\right|\left\{\left(1+\frac{|a|}{1 !}+\cdots+\frac{|a|^{n-s-1}}{(n-s-1) !}\right) \times \Omega(z)+\left(\frac{|a|^{n-s}}{(n-s) !}+\cdots+\frac{|a|^{n}}{n !}\right) \Omega(z)\right\} \\
& \leq\left|e^{a z}\right| e^{|a|}\left\{\chi+\frac{|a|^{n-s}}{(n-s) !}\right\} \Omega(z) .
\end{aligned}
$$

Using (2.21) we find, for $n \geq s+1$ and $|z| \geq r_{0}$

$$
\frac{\left|g^{(n)}(z)\right|}{n !} \leq e^{2|a|}\left(\chi+\frac{|a|^{n-s}}{(n-s) !}\right) \Omega^{* *}(z) .
$$

Since $s$ is fixed $|a|^{n-s} /(n-s) ! \rightarrow 0$ as $n \rightarrow \infty$, and so we may select, in view of (1.3), an integer $s_{0} \geq s$, so that

$$
e^{2|a|}\left(\chi+\frac{|a|^{n-s}}{(n-s) !}\right)<\chi^{\prime}<1
$$

as soon as $n \geq s_{0}+1$. Hence for all $n \geq s_{0}+1$ and all $z$ with $|z| \geq r_{0}$,

$$
\frac{\left|g^{(n)}(z)\right|}{n !} \leq \chi^{\prime} \Omega^{* *}(z) \leq \chi^{\prime} \max _{0 \leq j \leq s}\left\{\frac{\left|g^{(j)}(z)\right|}{j !}\right\} \text {. }
$$

The proof of Theorem 1 is now complete.

3. Lemmas. We require several lemmas. The first two lemmas contain known results.

Lemma A [2, Example B. 18]. If $\left\{b_{n}\right\}_{1}^{\infty}$ is positive nondecreasing and $\Sigma\left(n b_{n}\right)^{-1}<\infty$, then

$$
\lim _{n \rightarrow \infty} \frac{\log n}{b_{n}}=0
$$

Lemma $B\left[4\right.$, p. 261]. If $\alpha_{1} \leq \alpha_{2} \leq \cdots \leq a_{n^{\prime}}$ and $\beta_{1} \leq \beta_{2} \leq \cdots \leq \beta_{n}$, then 


$$
\alpha_{1} \beta_{j_{1}}+\alpha_{2} \beta_{j_{2}}+\cdots+a_{n} \beta_{j_{n}} \leq \alpha_{1} \beta_{1}+\alpha_{2} \beta_{2}+\cdots+\alpha_{n} \beta_{n}
$$

for every permutation $j_{1}, \cdots, j_{n}$ of $1,2, \cdots, n$.

Lemma 1. Let $a_{n+1}-a_{n} \geq b_{n}(n \geq 1)$ where $\left\{b_{n}\right\}_{1}^{\infty}$ is positive nondecreas. ing and $\Sigma\left(n b_{n}\right)^{-1}<\infty$. Given $K \geq 1$ and $\epsilon>0$, it is possible to find an integer $N \geq 1$ and a positive nondecreasing sequence $\left\{c_{n}\right\}_{n=1}^{\infty}$ such that the following conditions bold simultaneously:

$$
\begin{gathered}
c_{n} \geq 8 \quad(n \geq N), \\
\sum_{j=N}^{\infty} \frac{1}{j c_{j}}<\frac{\epsilon}{4}, \\
a_{n+1}-a_{n} \geq K c_{n}+8 \quad(n \geq N), \\
\sum_{j=1}^{\infty} \frac{1}{a_{j}}<+\infty, \\
\frac{1}{a_{N+2 m+1}-a_{N}>a_{N+2 m}-a_{N} \geq(K+1) m c_{N+m}} \quad(m \geq 1), \\
\frac{1}{c_{n+1}+\frac{1}{c_{n+1}+c_{n+2}}+\frac{1}{c_{n+1}+c_{n+2}+c_{n+3}}+\cdots \leq \epsilon \quad(n \geq N),} \\
\frac{1}{2 c_{n-2}}+\frac{1}{3 c_{n-3}}+\cdots+\frac{1}{(n-N) c_{N}}<\epsilon \quad(n>N) .
\end{gathered}
$$

Proof. (i) Let $c_{n}=b_{n} /(K+1)$. Then $\left\{c_{n}\right\}_{n=1}^{\infty}$ is the required sequence such that $\Sigma\left(n c_{n}\right)^{-1}<+\infty$. By Lemma $A$ and the convergence of this series we can choose $N$ so large that (3.3) and (3.4) are satisfied.

$$
a_{n+1}-a_{n} \geq b_{n}=(K+1) c_{n} \geq K c_{n}+8 \quad(n \geq N) .
$$

This proves (3.5).

(iii) Since

$$
a_{p+2 m}-a_{p}=\sum_{j=0}^{2 m-1}\left(a_{p+j+1}-a_{p+j}\right) \geq(K+1) \sum_{j=0}^{2 m-1} c_{p+j},
$$

we have on taking $p=2, p+2 m=2 n$,

$$
a_{2 n}>(K+1)\left(c_{n}+\cdots+c_{2 n-1}\right) \geq(K+1) n c_{n} .
$$

Hence

$$
\frac{1}{a_{2 n}}+\frac{1}{a_{2 n+1}}<\frac{2}{K+1} \frac{1}{n c_{n}}
$$


and the convergence of the series in (3.6) follows from (3.4).

(iv) Taking $p=N$ we get

$$
a_{N+2 m}-a_{N} \geq(K+1) m c_{N+m}
$$

Since $\left\{a_{n}\right\} \uparrow,(3.7)$ follows.

(v) We have

$$
c_{n+1}+\cdots+c_{n+2 m-1} \geq c_{n+m}+\cdots+c_{n+2 m-1} \geq m c_{n+m} \quad(m \geq 1)
$$

and sn

$$
\begin{aligned}
\sum_{n} & =\frac{1}{c_{n+1}}+\frac{1}{c_{n+1}+c_{n+2}}+\cdots \\
& \leq 2 \sum_{j=1}^{\infty} \frac{1}{j c_{n+j}}<\frac{2}{c_{n}} \sum_{j=1}^{n} \frac{1}{j}+2 \sum_{j=n+1}^{\infty} \frac{1}{j c_{n+j}} \\
& <\frac{2}{c_{n}}(1+\log n)+2 \sum_{j=n+1}^{\infty} \frac{1}{j c_{n+j}} .
\end{aligned}
$$

By Lemma A

$$
2(1+\log n) / c_{n}<\epsilon / 2 \text { if } n \geq N_{1}
$$

and

$$
2 \sum_{j=n+1}^{\infty} \frac{1}{j c_{n+j}}<\frac{\epsilon}{2} \text { if } n \geq N_{2} \text {. }
$$

Let $N=\max \left(N_{1}, N_{2}\right)$. Then for $n \geq N, \Sigma_{n}<\epsilon$ and (3.8) is proved.

(vi) Let $n>N$ and $r=1 / c_{n-1}+1 / 2 c_{n-2}+\cdots+1 /(n-N) c_{N^{*}}$ By (i),

(a)

$$
\frac{1}{c_{n-1}} \leq \frac{1}{c_{n-2}} \leq \cdots \leq \frac{1}{c_{N}},
$$

and

(b)

$$
\frac{1}{n-N}<\frac{1}{n-N-1}<\cdots<\frac{1}{2}<\frac{1}{1} .
$$

By applying Lemma $B$ to (a) and (b) we have

$$
\begin{aligned}
r & \leq \frac{1}{c_{N}}+\frac{1}{2 c_{N+1}}+\cdots+\frac{1}{(n-N) c_{n-1}} \\
& \leq \frac{1}{c_{N}}\left(1+\frac{1}{2}+\cdots+\frac{1}{N}\right)+\frac{1}{(N+1) c_{N+N}}+\cdots+\frac{1}{(n-N) c_{n-1}} \\
& \leq \frac{1+\log N}{c_{N}}+\sum_{j=1}^{\infty} \frac{1}{(N+j) c_{N+j}}<\epsilon
\end{aligned}
$$


for $N$ sufficiently large. This proves (3.9) and also completes the proof of Lemma 1.

In what follows in this section and in the next section we shall take $\epsilon=1 / 100$ and $N \geq 1$ such that (3.3)-(3.9) hold and also

$$
\frac{1}{c_{N}}+\sum_{j=1}^{\infty} \frac{1}{j c_{N+j}}<\frac{K}{100} \text {. }
$$

Lemma 2. Let $\Gamma_{n}=\left\{z:\left|z+a_{n}\right|<4\right\}, n \geq N$, and suppose $z \notin \bigcup_{n=N}^{\infty} \Gamma_{n}$. Then

$$
\sum_{j=N}^{\infty} \frac{1}{\left|z+a_{j}\right|}<\chi<1
$$

where $\chi$ is some fixed number.

Proof. Let $z=x+i y$. Then either

(i) $x \geq-a_{N}$, or

(ii) $-a_{n+1} \leq x<-a_{n}$ for some well-determined $n \geq N$.

Suppose first that (i) holds. By assumption

$$
1 /\left|z+a_{N}\right| \leq 1 / 4
$$

and

$$
\left|z+a_{N+1}\right| \geq\left|x+a_{N+1}\right| \geq a_{N+1}-a_{N} \geq(K+1) c_{N} .
$$

Hence

(iv)

$$
1 /\left|z+a_{N+1}\right| \leq 1 /(K+1) c_{N}<1 / 100
$$

For $j \geq N+2$,

$$
\left|z+a_{j}\right| \geq\left|x+a_{j}\right| \geq a_{j}-a_{N}
$$

Hence by (3.7) we have for $j=N+2 m$ or $N+2 m+1, m \geq 1$,

$$
\left|z+a_{j}\right| \geq(K+1) m c_{N+m}
$$

\section{Consequently}

$$
\sum_{j=N+2}^{\infty} \frac{1}{\left|z+a_{j}\right|} \leq \frac{1}{K+1} \sum_{m=1}^{\infty} \frac{1}{m c_{N+m}}<\frac{1}{100}
$$

and (3.10) follows from (iii)-(v). Suppose now (ii) holds. Then

$$
1 /\left|z+a_{n}\right|+1 /\left|z+a_{n+1}\right|<1 / 2
$$

For $j \geq n+2$,

$$
\left|z+a_{j}\right| \geq\left|x+a_{j}\right| \geq a_{j}-a_{n+1} \geq(K+1)\left(c_{j-1}+c_{j-2}+\cdots+c_{n+1}\right)
$$


and so, by (3.8),

(vii)

$$
\sum_{j=n+2}^{\infty} \frac{1}{\left|z+a_{j}\right|}<\frac{1}{K+1}\left\{\frac{1}{c_{n+1}}+\frac{1}{c_{n+1}+c_{n+2}}+\cdots\right\}<\frac{1}{100} .
$$

If $n>N$ we have for $N \leq j \leq(n-1)$

$$
\left|z+a_{j}\right| \geq\left|x+a_{j}\right| \geq\left|a_{n}-a_{j}\right| \geq(K+1)(n-j) c_{j}
$$

and (3.9) yields

(viii) $\sum_{j=N}^{n-1} \frac{1}{\left|z+a_{j}\right|} \leq \frac{1}{K+1}\left\{\frac{1}{c_{n-1}}+\frac{1}{2 c_{n-2}}+\cdots+\frac{1}{(n-N) c_{N}}\right\}<\frac{1}{10 n}$.

From (vi)-(viii) we get (3.10) in this case also. The proof of Lemma 2 is complete.

Lemma 3. Let

$$
f(z)=\prod_{j=N}^{\infty}\left(1+\frac{z}{a_{j}}\right)
$$

and let $\left\{-d_{j}\right\}_{j=N}^{\infty}$ be the zeros of $f^{\prime}(z)$. Then for all $j$ and $k(j \geq N, k \geq N)$,

$$
\left|d_{j}-a_{k}\right|>8 \text {. }
$$

Proof. We need to show that if $\left|z+a_{k}\right| \leq 8$ for some $k$, then

$$
\frac{f^{\prime}(z)}{f(z)}=\sum_{j=N}^{\infty} \frac{1}{z+a_{j}} \neq 0 \text {. }
$$

For $j \geq k+1$, we have by (3.5),

$$
\left|z+a_{j}\right| \geq\left|a_{j}-a_{k}\right|-\left|z+a_{k}\right| \geq\left(a_{j}-a_{k}\right)-8 \geq K\left(c_{j-1}+c_{j-2}+\cdots+c_{k}\right) .
$$

Hence by (3.8)

$$
\sum_{j=k+1}^{\infty} \frac{1}{\left|z+a_{j}\right|}<\frac{1}{K}\left\{\frac{1}{c_{k}}+\frac{1}{c_{k}+c_{k+1}}+\cdots\right\}<\frac{1}{50} .
$$

If $k>N$ we have for $N \leq j \leq k-1$ (see (3.5)),

$$
\left|z+a_{j}\right| \geq\left(a_{k}-a_{j}\right)-\left|z+a_{k}\right| \geq a_{k}-a_{j}-8 \geq K(k-j) c_{j}
$$

This gives, by (3.9),

$$
\sum_{j=N}^{k-1} \frac{1}{\left|z+a_{j}\right|} \leq \frac{1}{K}\left(\frac{1}{c_{k-1}}+\frac{1}{2 c_{k-2}}+\cdots+\frac{1}{(k-N) c_{N}}\right)<\frac{1}{100} .
$$

Since $\left|z+a_{k}\right| \leq 8$, (i) and (ii) imply

$$
\frac{f^{\prime}(z)}{f(z)} \geq \frac{1}{8}-\frac{1}{100}-\frac{1}{50}>0 \text { 。 }
$$


This completes the proof of Lemma 3.

Lemma 4. If $z \in \Gamma_{n}=\left\{z:\left|z+a_{n}\right|<4\right\}$ for some $n \geq N$, then

$$
\sum_{j=N}^{\infty} \frac{1}{\left|z+d_{j}\right|}<\chi<1
$$

where $\chi$ is some fixed number.

Proof. (a) We have, by Laguerre's theorem [1, p. 23], $a_{N}<d_{N}<a_{N+1}<\cdots$. Suppose first

$$
\left|z+a_{N}\right|<4
$$

By (3.11) and (i)

$$
\left|z+d_{N}\right| \geq\left(d_{N}-a_{N}\right)-\left|z+a_{N}\right|>8-4=4 .
$$

For $j \geq N+1$ we have by (3.5)

$$
\left|z+d_{j}\right| \geq\left(d_{j}-a_{N}\right)-\left|z+a_{N}\right| \geq a_{j}-a_{N}-4 \geq K\left\{c_{j-1}+c_{j-2}+\cdots+c_{N}\right\} .
$$

Hence by (3.8),

$$
\sum_{j=N+1}^{\infty} \frac{1}{\left|z+d_{j}\right|}<\frac{1}{K}\left\{\frac{1}{c_{N}}+\frac{1}{c_{N}+c_{N+1}}+\cdots\right\}<\frac{1}{50} .
$$

These two inequalities (ii) and (iii) give (3.12).

(b) Suppose now $\left|z+a_{n}\right|<4$ for some $n \geq N+1$. Then

$$
\left|z+d_{n}\right| \geq\left(d_{n}-a_{n}\right)-\left|z+a_{n}\right| \geq 8-4=4 \text {. }
$$

Similarly $\left|z+d_{n-1}\right|>4$ and so

$$
1 /\left|z+d_{n-1}\right|+1 /\left|z+d_{n}\right|<1 / 2
$$

For $j \geq n+1$, we have by (3.5)

$$
\left|z+d_{j}\right| \geq\left(d_{j}-a_{n}\right)-\left|z+a_{n}\right|>\left(a_{j}-a_{n}\right)-4 \geq K\left(c_{j-1}+c_{j-2}+\cdots+c_{n}\right) .
$$

This gives (see (3.8))

(v)

$$
\sum_{j=n+1}^{\infty} \frac{1}{\left|z+d_{j}\right|}<\frac{1}{K}\left\{\frac{1}{c_{n}}+\frac{1}{c_{n}+c_{n+1}}+\cdots\right\}<\frac{1}{100} .
$$

If $N \leq n-2$ we have for $N \leq j \leq n-2$,

$$
\left|z+d_{j}\right| \geq\left|d_{j}-a_{n}\right|-\left|z+a_{n}\right| \geq a_{n}-a_{j+1}-4 \geq K(n-j-1) c_{j+1} .
$$

Hence (3.9) yields 
(vi) $\sum_{j=N}^{n-2} \frac{1}{\left|z+d_{j}\right|}<\frac{1}{K}\left\{\frac{1}{c_{n-1}}+\frac{1}{2 c_{n}}+\cdots+\frac{1}{(n-N-1) c_{N+1}}\right\}<\frac{1}{100}$.

The inequalities (iv)-(vi) imply (3.12). The proof of Lemma 4 is complete.

Lemma 5. Let

$$
f(z)=\prod_{j=N}^{\infty}\left(1+\frac{z}{a_{j}}\right)
$$

Then $f(z)$ is an entire function of genus zero and

$$
f^{\prime}(z)=f^{\prime}(0) \prod_{j=N}^{\infty}\left(1+\frac{z}{d_{j}}\right)
$$

where $f^{\prime}(0)=\sum_{j=N}^{\infty} a_{j}^{-1}$. If for some $z$ at least one of the two inequalities

$$
\sum_{j=N}^{\infty} \frac{1}{\left|z+a_{j}\right|}<\chi<1
$$

(ii)

$$
\sum_{j=N}^{\infty} \frac{1}{\left|z+d_{j}\right|}<\chi<1
$$

where $\chi$ is a constant, bolds, then for this $z$

$$
\begin{aligned}
\frac{\left|f^{(n+1)}(z)\right|}{(n+1) !} & \leq \max \left\{\chi^{n+1}|f(z)|, \frac{\chi^{n}}{(n+1)}\left|f^{\prime}(z)\right|\right\} \\
& <\chi^{n} \max \left\{|f(z)|,\left|f^{\prime}(z)\right|\right\}, \quad n=1,2, \ldots
\end{aligned}
$$

Proof. Let

$$
p(z)=\sum_{j=N}^{\infty} \frac{1}{\left(z+a_{j}\right)}
$$

and suppose (i) of (3.13) holds. Then

(iii)

$$
|p(z)|<x<1,
$$

Hence

$$
p^{(n)}(z)=(-1)^{n} n ! \sum_{j=N}^{\infty} \frac{1}{\left(z+a_{j}\right)^{n+1}}, \quad n=1,2, \ldots
$$

(iv)

$$
\frac{\left|p^{(n)}(z)\right|}{n !} \leq \sum_{j=N}^{\infty} \frac{1}{\left|z+a_{j}\right|^{n+1}} \leq\left(\sum_{j=N}^{\infty} \frac{1}{\left|z+a_{j}\right|}\right)^{n+1}<\chi^{n+1} .
$$

Since $f^{\prime}=f p$ we have

$$
\left|f^{\prime}(z)\right| \leq \chi|f(z)|
$$

and 


$$
\frac{\left|f^{(n+1)}(z)\right|}{(n+1) !}=\frac{1}{n+1}\left|\left\{\frac{f^{(n)}(z)}{n !} \frac{p(z)}{0 !}+\frac{f^{(n-1)}(z)}{(n-1) !} \frac{p^{\prime}(z)}{1 !}+\cdots+\frac{f(z)}{0 !} \frac{p^{(n)}(z)}{n !}\right\}\right|
$$

$$
\leq \frac{1}{n+1}\left\{\frac{\left|f^{(n)}(z)\right|}{n !} \chi+\frac{\left|f^{(n-1)}(z)\right|}{(n-1) !} \chi^{2}+\cdots+|f(z)| \chi^{n+1}\right\} \text {. }
$$

We now use an induction argument to show that

$$
\frac{\left|f^{(n)}(z)\right|}{n !} \leq \chi^{n}|f(z)|, \quad n=1,2, \ldots
$$

For the inequality holds by (v) for $n=1$. Suppose it is true for $n=1,2, \cdots$, $m$. Then by (vi),

$$
\frac{\left|f^{(m+1)}(z)\right|}{(m+1) !} \leq \frac{1}{m+1}\left\{\frac{f^{(m)}(z)}{m !} \chi+\cdots+|f(z)| \chi^{m+1}\right\}
$$

$$
<\frac{1}{m+1}\left\{\chi^{m} \chi+\chi^{m-1} \chi^{2}+\cdots+\chi^{m+1}\right\}|f(z)|=\chi^{m+1}|f(z)| \text {. }
$$

This proves (vii). Suppose now (ii) of (3.13) holds. We have then

$$
f^{\prime \prime}(z)=f^{\prime}(z) \sum_{j=N}^{\infty} \frac{1}{\left(z+d_{j}\right)}
$$

and the above reas oning yields

$$
\frac{\left|f^{(n+2)}(z)\right|}{(n+1) !}<\chi^{n+1}\left|f^{\prime}(z)\right| \quad(n=0,1,2, \ldots) .
$$

From (vii) and (ix) we have

$$
\begin{aligned}
\frac{\left|f^{(n+1)}(z)\right|}{(n+1) !} & \leq \max \left\{\chi^{n+1}|f(z)|, \frac{\chi^{n}}{n+1}\left|f^{\prime}(z)\right|\right\} \\
& <\chi^{n} \max \left\{|f(z)|,\left|f^{\prime}(z)\right|\right\} \quad(n=1,2, \ldots) .
\end{aligned}
$$

This completes the proof of Lemma 5 .

4. Proof of Theorem 2. We have

$$
f(z)=\prod_{j=1}^{\infty}\left(1+\frac{z}{a_{j}}\right)=\prod_{1}^{N-1}\left(1+\frac{z}{a_{j}}\right) \prod_{j=N}^{\infty}\left(1+\frac{z}{a_{j}}\right)=P(z) f_{N}(z),
$$

where $P(z)$ is a polynomial of degree $(N-1)$ and $f_{N}(z)=\prod_{j=N}^{\infty}\left(1+z / a_{j}\right)$ and $N$ is determined as in the remark following Lemma 1.

Let $z$ be given. Then either $z \in \Gamma_{n}$ for some $n \geq N$ or $z \notin \bigcup_{n=N}^{\infty} \Gamma_{n}$. If $z \in \Gamma_{n}$ for some $n \geq N$, then by Lemma 4, (ii) of (3.13) holds and hence, by Lemma $5,(3.14)$ holds with $f$ replaced by $f_{N}$.

If $z \notin \bigcup_{n=N}^{\infty} \Gamma_{n}$, then by Lemma 2, (i) of (3.13) holds and we have, again by Lemma 5, (3.14) with $f$ replaced by $f_{N}$. Hence $f_{N} \in S B$ and so by Theorem 1 , 
$f(z)=P(z) f_{N}(z)$ belongs to $S B$. This completes the proof of Theorem 2 .

The corollaries are almost obvious and we leave the proofs to the reader. Note that if $f(\alpha z) \in S B$ then $f(|\alpha| z) \in S B$ and conversely..

\section{REFERENCES}

1. R. P. Boas, Jr., Entire functions, Academic Press, New York, 1954. MR 16, 914.

2. E. C. Francis and J. E. Littlewood, Examples in infinite series with solutions, Deighton, Cambridge, 1953.

3. Fred Gross, Entire functions of bounded index, Proc. Amer. Math. Soc. 18 (1967), 974-980. MR 36 \#1649.

4. G. H. Hardy, J. E. Littlew ood and G. Pólya, Inequalities, Cambridge Univ. Press, New York, 1964.

5. Boo Sang Lee and S. M. Shah, On the growth of entire functions of bounded index, Indiana Univ. Math. J. 20 (1970/71), 81-87. MR 41 \#3766.

6. Benjamin Lepson, Differential equations of infinite order hyperdirichlet series and entire functions of bounded index, Proc. Sympos. Pure Math., vol. 11, Amer. Math. Soc., Providence, R. I., 1968, pp. 298-307. MR $38 \# 6069$.

7. Walter Pugh and S. M. Shah, On the growth of entire functions of bounded index, Pacific J. Math. 33 (1970), 191-201. MR 41 \#3767.

8. S. M. Shah, Entire functions of bounded index, Proc. Amer. Math. Soc. 19 (1968), 1017-1022. MR 38 \#6070.

9. - Entire functions satisfying a linear differential equation, J. Math. Mech. 18 (1968/69), 131-136. MR 37 \#2994.

10. - On entire functions of bounded index whose derivatives are of unbounded index, J. London Math. Soc. (2) 4 (1971), 127-139.

DEPARTMENT OF MATHEMATICS, UNIVERSITY OF KENTUCKY, LEXINGTON, KENTUCKY 40506 (Current 2ddress of S. M. Shah)

DEPARTMENT OF MATHEMATICS, SYRACUSE UNIVERSITY, SYRACUSE, NEW YORK 13210

Current address (S. N. Shah): Department of Mathematics, Hampton Institute, Hampton, Virginia 23368 